\title{
IDENTIFICACIÓN Y ANÁLISIS DE LOS H-CLASSICS DE LAS UNIVERSIDADES PÚBLICAS ANDALUZAS EN LA CATEGORÍA ECONOMICS DEL JOURNAL CITATION REPORTS (JCR).
}

\section{IDENTIFICATION AND ANALYSIS OF THE H- CLASSICS OF ANDALUSIAN PUBLIC UNIVERSITIES IN THE ECONOMICS CATEGORY OF THE JOURNAL CITATION REPORTS (JCR).}

Jesús Cascón Katchadourian (Universidad de Granada). ${ }^{1}$

\section{Resumen:}

Los artículos altamente citados muestran una perspectiva de aquellos documentos que, históricamente, han atraído un gran interés por parte de una comunidad investigadora y que se podrían considerar como la base del campo de investigación. En el año 2014 se desarrolló un nuevo enfoque para identificar estos artículos altamente citados que se llama H-Classics. Se basa en el índice $\mathrm{H}$ y refleja tanto a las características propias de la disciplina de investigación correspondiente como su evolución. El presente estudio tiene como objetivo identificar y analizar los $\mathrm{H}$-Classics de los artículos publicados por investigadores de las universidades públicas andaluzas, dentro de la categoría Economics del Journal Citation Reports. La metodología utilizada es el análisis bibliométrico a través de la técnica del H-Classics con la cual se extraen los principales indicadores de rendimiento. Este análisis proporciona unos resultados que nos dan una visión útil sobre el desarrollo de este campo de investigación, revelando aquellos actores científicos que han realizado la mayor contribución a su desarrollo: autores, revistas, áreas de conocimiento relacionadas, países, universidades andaluzas, universidades nacionales y extranjeras. Se concluye el artículo con las limitaciones de este y los principales hallazgos encontrados.

Palabras clave: H-Index, H-Classics, bibliometría, Economics, universidades públicas andaluzas.

Códigos JEL: C00.

\section{Abstract:}

Highly Cited Papers offer an outlook on those papers that have attracted great historical interest by a research community and that could also be considered the basis of the research field. In 2014 a new approach was recognised to identify these highly cited articles called H-Classics. It is based on the $\mathrm{H}$-index and is sensitive to both the characteristics of the corresponding research discipline and its evolution. The objective of this study is to identify and analyze the H-Classics of articles published by researchers from Andalusian public universities, within the Economics category of the Journal Citation Reports. The methodology used is the bibliometric analysis through the H-Classics technique with which the main performance indicators are extracted. This analysis provides results that give us a useful insight into the development of this field of

\footnotetext{
${ }^{1}$ cascon@ugr.es, Universidad de Granada.

Recibido: 31 de marzo de 2020. Aceptado: 21 de mayo de 2020.
} 
research, revealing those scientific actors who have made the greatest contribution to its development: authors, journals, related areas of knowledge, countries, Andalusian universities, national and foreign universities. The article is concluded with its limitations and the main findings found.

Keywords: H-Index, H-Classics, bibliometrics, Economics, Andalusian public universities. JEL Codes: C00.

\section{INTRODUCCIÓN.}

En el año 1977 Eugene Garfield (1977) introdujo el concepto bibliométrico de "citation classic", también llamado "classic article" o "literacy classic" el cual permitía designar los artículos científicos altamente citados de una determinada disciplina. La principal virtud de la aplicación de este concepto es que ayuda a entender el desarrollo de una disciplina y, por tanto, el pasado, presente y futuro de la estructura científica de un campo del saber (Martínez et al., 2014a). Los artículos altamente citados son importantes porque atraen el interés de la comunidad científica y, por ello, son considerados los "lingotes de oro de la ciencia" (Smith, 2007; Stack, 2012). Una vez identificados, su lectura y análisis nos ayudan a conocer los mayores avances de una determinada disciplina, los temas de investigación más candentes y activos y los principales actores del campo de investigación, como pueden ser las revistas, científicos, países, grupos de investigación o instituciones (Baltussen y Kindler, 2004; Garfield, 1977; Smith, 2007; Stack, 2012; Tam et al., 2013).

Debido a tales virtudes, se han publicado un buen número de estudios que tratan sobre artículos altamente citados o sobre citation classics en distintas disciplinas. Algunos ejemplos destacables en diversas áreas de conocimiento son "Integrative \& Complementary Medicine" (Tam et al., 2012), "Neurosurgery” (Ponce y Lozano, 2010), "Parkinson” (Ponce y Lozano, 2011), "Critical Care Medicine” (Rosenberg et al., 2010), "Suicidology” (Stack, 2012), "Deviant Behavior" (Stack, 2013), "Information \& Library Science” (Levitt and Thelwall, 2009), “Occupational Medicine” (Gehanno et al., 2007), "Epilepsy” (Ibrahim et al., 2012), “ Dentistry” (Feijoo et al., 2013), “Orthodontist” (Hui et al., 2013), “Arthroscopy” (Cassar et al., 2012), "Implantology" (Chiang et al., 2018) Prominence of scientific publications towards periimplant complications in implantology: A bibliometric analysis using the H-classics method, 2018) “Group decision making” (Cabrerizo et al., 2017), y "Rheumatology” (Perez-Cabezas et al., 2018).

Una característica común de este tipo de estudios es fijar como criterio un valor umbral que distinga los artículos muy citados de los que no lo son; para ello se siguen las recomendaciones de Garfield (Garfield, 1977, 1987). En tal sentido, existen dos enfoques: (Martínez et al., 2014b): uno, fijación del valor umbral en las citas recibidas (Gehanno et al., 2007; Ibrahim et al., 2012; Ponce y Lozano, 2010, 2011; Rosenberg et al., 2010); y otro, establecimiento del valor umbral según el número de documentos muy citados a recuperar (Cassar et al., 2012; Feijoo et al., 2013; Hui et al., 2013; Levitt y Thelwall, 2009; Stack, 2012, 2013; Tam et al., 2012).

El problema de estos enfoques es que no están basados en algo objetivo y surgen preguntas que no tienen respuesta si usamos estos métodos. ¿Por qué se utiliza como umbral 400 citas, o 100 o 2.000 y no otra cantidad parecida? ¿Por qué nos quedamos solo con el top 1 por cien y no con el 3 por cien o el 5 por cien? 
Además, ambos enfoques no tienen en cuenta los patrones de citas y la evolución científica de las áreas de investigación. Para superar esos problemas Martínez et al. (2014b) presentaron el concepto de H-Classics basado en el popular índice H (Hirsch, 2005), que proporciona un criterio objetivo e imparcial para construir los artículos altamente citados para cualquier campo de investigación.

En nuestro trabajo se presenta un análisis de los artículos altamente citados de entre los producidos por los investigadores de universidades públicas andaluzas en el área "Economics" del Web of Science (WoS) utilizando el H-Classics. El objetivo es ofrecer una panorámica de la estructura científica del campo.

El resto del documento está organizado de la siguiente manera: el apartado 2 muestra la metodología utilizada en el análisis. El apartado 3 presenta los resultados de nuestro análisis. Finalmente, las conclusiones se muestran en el apartado 4.

\section{METODOLOGÍA.}

\subsection{Fuente de los datos.}

En este estudio se ha utilizado la base de datos Web of Science (WoS) de Clarivate Analytics. Se ha elegido esta base de datos entre las existentes como Scopus o Google Scholar por ser la base de datos líder mundial sobre producción científica con una mayor cobertura tanto actual como como en el pasado llegando al año 1900, superando en este apartado a Scopus (Martínez et al., 2015). Además, nos ofrece más seguridad que Google Scholar por varios motivos, esta última es menos rigurosa e incorpora publicaciones no científicas (Martínez et al., 2015), y no funciona bien con estudios retrospectivos pues las publicaciones antiguas no están todavía colgadas en la web (Harzing y van der Wal, 2008, p. 65). Por último, la WoS incorpora una gran cantidad de herramientas de análisis para procesado de datos que se utilizan en este estudio.

\subsection{Ecuación y procedimiento de búsqueda.}

Se ha utilizado la búsqueda avanzada por el campo Web of Science Categories (WC), la categoría "economics", como tipos de documentos se han escogido Article y Review. Una vez realizada la búsqueda se ha filtrado por país España y en segundo lugar por organización consolidada, eligiendo a las 9 universidades públicas andaluzas. Se han escogido todas las bases de datos y como periodo de tiempo todos los años disponibles. Se ha escogido esta categoría en consonancia con lo que se quiere hacer en este estudio reflejado en el título; article y review por ser los tipos documentales más importantes y los utilizados para obtener los H-Classics; el filtrado ha sido el adecuado para obtener sólo resultados donde haya autores de las universidades públicas andaluzas; igualmente se han escogido todas las bases de datos para incorporar todos los artículos posibles y no sólo el core; y todos los años disponibles porque queremos saber los H-Classics de toda la historia y no sólo de un marco temporal concreto.

Está búsqueda, realizada el 05 de marzo de 2020, nos da un total de 1787 documentos.

\subsection{Cálculo del índice $\mathrm{H}$ del conjunto de documentos.}

El índice-H es uno de los principales indicadores bibliométricos de la producción y la calidad científica; fue diseñado por Hirsch (Hirsch, 2005) para medir el rendimiento de los investigadores (Alonso y otros, 2009). El procedimiento para calcular el índice H de un autor, una revista, campo de investigación o de un conjunto de documentos es siempre el mismo; en primer lugar, es necesario establecer una clasificación de los documentos de acuerdo con sus 
citas, es decir, el conjunto de documentos debe ordenarse por citas de forma decreciente, teniendo el documento con más citas la posición número 1, el segundo con más citas la 2 y así sucesivamente.

En segundo lugar, hay que ubicar el primer documento cuya posición en la clasificación está por debajo de su recuento de citas. El índice H será la posición de clasificación del documento inmediatamente anterior. Aunque el índice $\mathrm{H}$ puede calcularse manualmente, como se mencionó anteriormente, hay diferentes herramientas disponibles en WoS que facilitan su cálculo para un conjunto dado de documentos; en la interfaz usada en este estudio la herramienta es "crear informe de citas". El índice H de los 1787 documentos es 50, es decir, el H-Classic de esos 1787 documentos son los 50 más citados. Estos documentos ordenados por citas de mayor a menor, con la referencia completa, se listan en la tabla 1.

\begin{tabular}{|c|c|}
\hline \multicolumn{2}{|l|}{ TABLA 1: ARTÍCULOS H-CLASSICS. } \\
\hline REFERENCIAS & CITAS \\
\hline $\begin{array}{l}\text { Dietsch, M., \& Lozano-Vivas, A. (2000, Jun). How the environment determines } \\
\text { banking efficiency: A comparison between French and Spanish industries. Journal of } \\
\text { Banking \& Finance, 24(6), 985-1004. https://doi.org/10.1016/s0378-4266(99)00115- } \\
6\end{array}$ & 195 \\
\hline $\begin{array}{l}\text { Delcastillo, J. M., \& Benitez, F. G. (1995a, Oct). ON THE FUNCTIONAL FORM } \\
\text { OF THE SPEED-DENSITY RELATIONSHIP .1. GENERAL-THEORY. } \\
\text { Transportation Research Part B-Methodological, 29(5), 373-389. }\end{array}$ & 164 \\
\hline $\begin{array}{l}\text { Lozano, S., \& Villa, G. (2004, Jul-Sep). Centralized resource allocation using data } \\
\text { envelopment analysis. Journal of Productivity Analysis, 22(1-2), 143-161. } \\
\text { https://doi.org/10.1023/b:prod.0000034748.22820.33 }\end{array}$ & 137 \\
\hline $\begin{array}{l}\text { Valverde, S. C., \& Fernandez, F. R. (2007, Jul). The determinants of bank margins in } \\
\text { European banking. Journal of Banking \& Finance, 31(7), 2043-2063. } \\
\text { https://doi.org/10.1016/j.jbankfin.2006.06.017 }\end{array}$ & 136 \\
\hline $\begin{array}{l}\text { De Ona, J., de Ona, R., Eboli, L., \& Mazzulla, G. (2013, Sep). Perceived service } \\
\text { quality in bus transit service: A structural equation approach. Transport Policy, 29, } \\
\text { 219-226. https://doi.org/10.1016/j.tranpol.2013.07.001 }\end{array}$ & 125 \\
\hline $\begin{array}{l}\text { Lozano-Vivas, A., Pastor, J. T., \& Pastor, J. M. (2002, Jul). An efficiency comparison } \\
\text { of European banking systems operating under different environmental conditions. } \\
\text { Journal of Productivity Analysis, 18(1), 59-77. } \\
\text { https://doi.org/10.1023/a:1015704510270 }\end{array}$ & 122 \\
\hline $\begin{array}{l}\text { Linan, F., \& Santos, F. J. (2007, Nov). Does Social Capital Affect Entrepreneurial } \\
\text { Intentions? International Advances in Economic Research, 13(4), 443-453. } \\
\text { https://doi.org/10.1007/s11294-007-9109-8 }\end{array}$ & 118 \\
\hline $\begin{array}{l}\text { Faria, R., Gomes, M., Epstein, D., \& White, I. R. (2014, Dec). A Guide to Handling } \\
\text { Missing Data in Cost-Effectiveness Analysis Conducted Within Randomised } \\
\text { Controlled Trials. Pharmacoeconomics, 32(12), 1157-1170. } \\
\text { https://doi.org/10.1007/s40273-014-0193-3 }\end{array}$ & 117 \\
\hline $\begin{array}{l}\text { Lozano, S., \& Gutierrez, E. (2008, Jul). Non-parametric frontier approach to } \\
\text { modelling the relationships among population, GDP, energy consumption and CO2 } \\
\text { emissions. Ecological Economics, 66(4), 687-699. } \\
\text { https://doi.org/10.1016/j.ecolecon.2007.11.003 }\end{array}$ & 114 \\
\hline $\begin{array}{l}\text { Lozano-Vivas, A., \& Pasiouras, F. (2010, Jul). The impact of non-traditional } \\
\text { activities on the estimation of bank efficiency: International evidence. Journal of } \\
\text { Banking \& Finance, 34(7), 1436-1449. https://doi.org/10.1016/j.jbankfin.2010.01.006 }\end{array}$ & 107 \\
\hline $\begin{array}{l}\text { Carbo-Valverde, S., Rodriguez-Fernandez, F., \& Udell, G. F. (2009, Apr). Bank } \\
\text { Market Power and SME Financing Constraints*. Review of Finance, 13(2), 309-340. } \\
\text { https://doi.org/10.1093/rof/rfp003 }\end{array}$ & 104 \\
\hline
\end{tabular}


Velazquez, E. (2006, Feb). An input-output model of water consumption: Analysing intersectoral water relationships in Andalusia. Ecological Economics, 56(2), 226-240. https://doi.org/10.1016/j.ecolecon.2004.09.026

Brouwer, R., Martin-Ortega, J., \& Berbel, J. (2010, Aug). Spatial Preference Heterogeneity: A Choice Experiment. Land Economics, 86(3), 552-568.

https://doi.org/10.3368/le.86.3.552

Gomez-Limon, J. A., \& Riesgo, L. (2004, Jul). Irrigation water pricing: differential impacts on irrigated farms. Agricultural Economics, 31(1), 47-66.

https://doi.org/10.1016/j.agecon.2003.02.001

Iniesta-Arandia, I., Garcia-Llorente, M., Aguilera, P. A., Montes, C., \& MartinLopez, B. (2014, Dec). Socio-cultural valuation of ecosystem services: uncovering the links between values, drivers of change, and human well-being. Ecological Economics, 108, 36-48. https://doi.org/10.1016/j.ecolecon.2014.09.028

Romero-Avila, D. (2008a, Sep). Convergence in carbon dioxide emissions among industrialised countries revisited. Energy Economics, 30(5), 2265-2282.

https://doi.org/10.1016/j.eneco.2007.06.003

Benavides-Velasco, C. A., Quintana-Garcia, C., \& Guzman-Parra, V. F. (2013, Jan). Trends in family business research. Small Business Economics, 40(1), 41-57. https://doi.org/10.1007/s11187-011-9362-3

Dietzenbacher, E., \& Velazquez, E. (2007, Apr). Analysing Andalusian virtual water trade in an input - Output framework. Regional Studies, 41(2), 185-196.

https://doi.org/10.1080/00343400600929077

Zahra, S. A., Van de Velde, E., \& Larraneta, B. (2007, Aug). Knowledge conversion capability and the performance of corporate and university spin-offs. Industrial and Corporate Change, 16(4), 569-608. https://doi.org/10.1093/icc/dtm018

del Rio, P., \& Gual, M. A. (2007, Feb). An integrated assessment of the feed-in tariff system in Spain. Energy Policy, 35(2), 994-1012.

https://doi.org/10.1016/j.enpol.2006.01.014

Cummins, J. D., \& Rubio-Misas, M. (2006, Mar). Deregulation, consolidation, and efficiency: Evidence from the Spanish insurance industry. Journal of Money Credit and Banking, 38(2), 323-355. https://doi.org/10.1353/mcb.2006.0029

Capra, C. M., Goeree, J. K., Gomez, R., \& Holt, C. A. (1999, Jun). Anomalous behavior in a traveler's dilemma? American Economic Review, 89(3), 678-690. https://doi.org/10.1257/aer.89.3.678

Millan, J. M., Hessels, J., Thurik, R., \& Aguado, R. (2013, Apr). Determinants of job satisfaction: a European comparison of self-employed and paid employees. Small Business Economics, 40(3), 651-670. https://doi.org/10.1007/s11187-011-9380-1

Cansino, J. M., Roman, R., \& Ordonez, M. (2016, Feb). Main drivers of changes in CO2 emissions in the Spanish economy: A structural decomposition analysis. Energy Policy, 89, 150-159. https://doi.org/10.1016/j.enpol.2015.11.020

Kumbhakar, S. C., Lozano-Vivas, A., Lovell, C. A. K., \& Hasan, I. (2001, Feb). The effects of deregulation on the performance of financial institutions: The case of Spanish savings banks. Journal of Money Credit and Banking, 33(1), 101-120. https://doi.org/10.2307/2673874

Barrena, E., Canca, D., Coelho, L. C., \& Laporte, G. (2014, Dec). Single-line rail rapid transit timetabling under dynamic passenger demand. Transportation Research Part B-Methodological, 70, 134-150. https://doi.org/10.1016/j.trb.2014.08.013 Millan, J. M., Congregado, E., \& Roman, C. (2012, Feb). Determinants of selfemployment survival in Europe. Small Business Economics, 38(2), 231-258. https://doi.org/10.1007/s11187-010-9260-0

Delcastillo, J. M., \& Benitez, F. G. (1995b, Oct). ON THE FUNCTIONAL FORM OF THE SPEED-DENSITY RELATIONSHIP .2. EMPIRICAL-INVESTIGATION. Transportation Research Part B-Methodological, 29(5), 391-406. 
Machuca, J. A. D., \& Barajas, R. P. (2004, May). The impact of electronic data interchange on reducing bullwhip effect and supply chain inventory costs.

Transportation Research Part E-Logistics and Transportation Review, 40(3), 209-228.

https://doi.org/10.1016/j.tre.2003.08.001

Nelsena, R. B., Quesada-Molina, J. J., Rodriguez-Lallena, J. A., \& Ubeda-Flores, M. (2008, Apr). On the construction of copulas and quasi-copulas with given diagonal sections. Insurance Mathematics \& Economics, 42(2), 473-483.

https://doi.org/10.1016/j.insmatheco.2006.11.011

Romero-Avila, D., \& Strauch, R. (2008, Mar). Public finances and long-term growth

in Europe: Evidence from a panel data analysis. European Journal of Political

Economy, 24(1), 172-191. https://doi.org/10.1016/j.ejpoleco.2007.06.008

Salgadoa, P. P., Quintana, S. C., Pereirac, A. G., Ituarte, L. D., \& Pedregal-Mateos,

B. (2009, Feb). Participative multi-criteria analysis for the evaluation of water governance alternatives. A case in the Costa del Sol (Malaga). Ecological Economics, 68(4), 990-1005. https://doi.org/10.1016/j.ecolecon.2006.11.008

Branas-Garza, P., Cobo-Reyes, R., Espinosa, M. P., Jimenez, N., Kovarik, J., \&

Ponti, G. (2010, Jul). Altruism and social integration. Games and Economic

Behavior, 69(2), 249-257. https://doi.org/10.1016/j.geb.2009.10.014

Linan, F., Nabi, G., \& Krueger, N. (2013). BRITISH AND SPANISH

ENTREPRENEURIAL INTENTIONS: A COMPARATIVE STUDY. Revista De

Economia Mundial (33), 73-103.

Parra-Lopez, C., Calatrava-Requena, J., \& de-Haro-Gimenez, T. (2008, Feb). A

systemic comparative assessment of the multifunctional performance of alternative

olive systems in Spain within an AHP-extended framework. Ecological Economics,

64(4), 820-834. https://doi.org/10.1016/j.ecolecon.2007.05.004

Branas-Garza, P. (2006, Jul). Poverty in dictator games: Awakening solidarity.

Journal of Economic Behavior \& Organization, 60(3), 306-320.

https://doi.org/10.1016/j.jebo.2004.10.005

Cansino, J. M., Pablo-Romero, M. D., Roman, R., \& Yniguez, R. (2010, Oct). Tax

incentives to promote green electricity: An overview of EU-27 countries. Energy

Policy, 38(10), 6000-6008. https://doi.org/10.1016/j.enpol.2010.05.055

Cummins, J. D., Rubio-Misas, M., \& Zi, H. M. (2004, Dec). The effect of

organizational structure on efficiency: Evidence from the Spanish insurance industry.

Journal of Banking \& Finance, 28(12), 3113-3150.

https://doi.org/10.1016/j.jbankfin.2004.05.004

Linan, F., \& Fernandez-Serrano, J. (2014, Apr). National culture, entrepreneurship

and economic development: different patterns across the European Union. Small

Business Economics, 42(4), 685-701. https://doi.org/10.1007/s11187-013-9520-x

Rubio, G., Pomares, H., Rojas, I., \& Herrera, L. J. (2011, Jul-Sep). A heuristic

method for parameter selection in LS-SVM: Application to time series prediction.

International Journal of Forecasting, 27(3), 725-739.

https://doi.org/10.1016/j.ijforecast.2010.02.007

Carbo-Valverde, S., Rodriguez-Fernandez, F., \& Udell, G. F. (2016, Feb). Trade

Credit, the Financial Crisis, and SME Access to Finance. Journal of Money Credit and Banking, 48(1), 113-143. https://doi.org/10.1111/jmcb.12292

Gual, M. A., \& Norgaard, R. B. (2010, Feb). Bridging ecological and social systems

coevolution: A review and proposal. Ecological Economics, 69(4), 707-717.

https://doi.org/10.1016/j.ecolecon.2008.07.020

Moscato, P., \& Cotta, C. (2010). A Modern Introduction to Memetic Algorithms. In

M. Gendreau \& J. Y. Potvin (Eds.), Handbook of Metaheuristics, Second Edition

(Vol. 146, pp. 141-183). Springer. https://doi.org/10.1007/978-1-4419-1665-5_6

Blancas, F. J., Caballero, R., Gonzalez, M., Lozano-Oyola, M., \& Perez, F. (2010,

Sep). Goal programming synthetic indicators: An application for sustainable tourism 


\begin{tabular}{|l|r|}
\hline $\begin{array}{l}\text { in Andalusian coastal counties. Ecological Economics, 69(11), 2158-2172. } \\
\text { https://doi.org/10.1016/j.ecolecon.2010.06.016 }\end{array}$ & 52 \\
\hline $\begin{array}{l}\text { Gomez-Herrera, E. (2013, Jun). Comparing alternative methods to estimate gravity } \\
\text { models of bilateral trade. Empirical Economics, 44(3), 1087-1111. } \\
\text { https://doi.org/10.1007/s00181-012-0576-2 }\end{array}$ & 52 \\
\hline $\begin{array}{l}\text { Gomez-Limon, J. A., Arriaza, M., \& Berbel, J. (2002, Jul). Conflicting } \\
\text { implementation of agricultural and water policies in irrigated areas in the EU. Journal } \\
\text { of Agricultural Economics, 53(2), 259-281. https://doi.org/10.1111/j.1477- } \\
\text { 9552.2002.tb00020.x }\end{array}$ & \\
\hline $\begin{array}{l}\text { Romero-Avila, D. (2008b, Jan). Questioning the empirical basis of the environmental } \\
\text { Kuznets curve fo CO2: New evidence from a panel stationarity test robust to multiple } \\
\text { breaks and cross-dependence. Ecological Economics, 64(3), 559-574. } \\
\text { https://doi.org/10.1016/j.ecolecon.2007.03.011 }\end{array}$ & 52 \\
\hline $\begin{array}{l}\text { Fernandez, J. I. P., \& Rivero, M. S. (2009, Jun). Measuring tourism sustainability: } \\
\text { proposal for a composite index. Tourism Economics, 15(2), 277-296. } \\
\text { https://doi.org/10.5367/000000009788254377 }\end{array}$ & 51 \\
\hline $\begin{array}{l}\text { Branas-Garza, P. (2007, Aug). Promoting helping behavior with framing in dictator } \\
\text { games. Journal of Economic Psychology, 28(4), 477-486. } \\
\text { https://doi.org/10.1016/j.joep.2006.10.001 }\end{array}$ & 50 \\
\hline $\begin{array}{l}\text { Gonzalez-Gomez, F., \& Garcia-Rubio, M. A. (2008). Efficiency in the management } \\
\text { of urban water services. What have we learned after four decades of research?. } \\
\text { Hacienda Publica Espanola-Review of Public Economics (185), 39-67. }\end{array}$ & 50 \\
\hline
\end{tabular}

Fuente: Elaboración propia.

\section{RESULTADOS DEL ANÁLISIS.}

En esta sección se analiza el H-Core del conjunto de documentos. En concreto se hace un análisis de los autores con más artículos H-Classics publicados; las revistas con mayor número de artículos H-Classics publicados; las áreas de conocimiento relacionadas con los $\mathrm{H}$-Classics publicados; el número de artículos H-Classics publicados por año; los países que más han publicado $\mathrm{H}$-Classics con las universidades andaluzas; las universidades andaluzas que más artículos H-classics han publicado; y las universidades nacionales y extranjeras con las que más se ha colaborado.

\subsection{Autores con más artículos H-Classics Publicados.}

La tabla 2 muestra todos los autores que tienen dos o más documentos publicados entre los H-Classics. En ella se muestra así mismo el nombre del autor, la institución a la que pertenecía el autor en el momento en que se publicaron los artículos, así como el número de documentos $\mathrm{H}$-Classics y el porcentaje sobre el total que estos suponen. El autor Branas-Garza, por ejemplo, pertenecía a la Universidad de Granada cuando publicó los artículos, sin embargo, hoy en día pertenece a la Universidad Loyola. Destaca en primer lugar y como único autor con cuatro documentos, Lozano-Vivas, catedrática de la Universidad de Málaga, miembro del departamento de Teoría e Historia Económica, directora del grupo de investigación fundamentos microeconómicos. En segundo lugar, hay tres investigadores con tres documentos cada uno, Branas-Garza, de la Universidad de Granada, Linan de la Universidad de Sevilla y Romero-Avila de la Universidad Pablo de Olavide. Estos serían los referentes andaluces en la disciplina. Aunque con el orden modificado, las cuatro primeras universidades andaluzas en el apartado de "Universidades que más artículos H-Classics han publicado" coinciden con el de las Universidades de adscripción de estos 4 autores. 


\begin{tabular}{|c|c|c|}
\hline \multicolumn{3}{|c|}{ TABLA 2: AUTORES CON MÁS ARTÍCULOS PUBLICADOS. } \\
\hline AUTORES & INSTITUCIÓN & $\begin{array}{l}\text { H-CLASSICS } \\
(\%)\end{array}$ \\
\hline LOZANO-VIVAS A & Universidad de Málaga & $4(8 \%)$ \\
\hline BRANAS-GARZA P & Universidad de Granada & $3(6 \%)$ \\
\hline LINAN F & Universidad de Sevilla & $3(6 \%)$ \\
\hline ROMERO-AVILA D & Universidad Pablo de Olavide & $3(6 \%)$ \\
\hline BENITEZ FG & Universidad de Sevilla & $2(4 \%)$ \\
\hline BERBEL J & Universidad de Córdoba & $2(4 \%)$ \\
\hline CANSINO JM & Universidad de Sevilla & $2(4 \%)$ \\
\hline CARBO-VALVERDE S & $\begin{array}{l}\text { Universidad de Granada. University } \\
\text { of Wales, Bangor (Reino Unido). }\end{array}$ & $2(4 \%)$ \\
\hline CUMMINS JD & University Penn Wharton & $2(4 \%)$ \\
\hline DELCASTILLO JM & Universidad de Sevilla & $2(4 \%)$ \\
\hline GOMEZ-LIMON JA & Universidad de Córdoba & $2(4 \%)$ \\
\hline GUAL MA & Universidad Pablo de Olavide & $2(4 \%)$ \\
\hline LOZANO S & Universidad de Sevilla & $2(4 \%)$ \\
\hline MILLAN JM & Universidad de Huelva & $2(4 \%)$ \\
\hline $\begin{array}{l}\text { RODRIGUEZ- } \\
\text { FERNANDEZ F }\end{array}$ & Universidad de Granada & $2(4 \%)$ \\
\hline ROMAN R & Universidad de Sevilla & $2(4 \%)$ \\
\hline RUBIO-MISAS M & Universidad de Málaga & $2(4 \%)$ \\
\hline UDELL GF & University of Indiana & $2(4 \%)$ \\
\hline VELAZQUEZ E & Universidad Pablo de Olavide & $2(4 \%)$ \\
\hline
\end{tabular}

\subsection{Revistas con mayor número de documentos H-Classics publicados.}

Estimamos que puede ser de interes para los investigadores del área, andaluces o no, saber en qué revistas se están publicando los artículos más influyentes de sus colegas andaluces. En la tabla 3 y la figura 1 se muestran todas aquellas revistas científicas que han publicado dos o más artículos H-Classics; dicha tabla está obtenida mediante la herramienta de procesamiento "analizar resultados" de la WoS. En el primer lugar del listado se encuentra Ecological Economics, publicando ocho artículos (el 16 por cien de los H-Classics), el doble de los publicados en la segunda revista. Hay que relacionar este resultado con el que se ve en el apartado “Áreas de conocimiento relacionadas”, en el mismo ENVIRONMENTAL SCIENCES ECOLOGY; es el área relacionada donde más H-Classics se publican. Destacar también aquí Journal of Banking \& Finance y Small Business Economics, en segundo lugar con cuatro artículos cada una.

Haciendo una comparación con la misma área temática dentro del Journal Citation Reports, esta área comprende 363 revistas; Ecological Economics, Energy Policy y Transportation Research Part B Methodological se encuentran en el primer decil, Small Business Economics y Journal of Banking \& Finance se encuentra en el primer cuartil, mientras que Journal of Money Credit and Banking y Journal of Productivity Analysis se encuentran en el segundo cuartil. Con el matiz de que este estudio se está refiriendo al ámbito de las universidades andaluzas, refleja también lo que otros estudiosos han reseñado anteriormente, que no siempre las mejores revistas tienen los artículos más citados y que revistas de menor impacto tienen artículos altamente citados (Seglen, 1997). 


\section{TABLA 3: REVISTAS CON MAYOR NÚMERO DE DOCUMENTOS} PUBLICADOS.

\begin{tabular}{|l|l|l|}
\hline Títulos de revistas & Número de artículos & \% de 50 \\
\hline ECOLOGICAL ECONOMICS & 8 & $16 \%$ \\
\hline JOURNAL OF BANKING \& FINANCE & 4 & $8 \%$ \\
\hline SMALL BUSINESS ECONOMICS & 4 & $8 \%$ \\
\hline ENERGY POLICY & 3 & $6 \%$ \\
\hline $\begin{array}{l}\text { JOURNAL OF MONEY CREDIT AND } \\
\text { BANKING }\end{array}$ & 3 & $6 \%$ \\
\hline $\begin{array}{l}\text { TRANSPORTATION RESEARCH PART B } \\
\text { METHODOLOGICAL }\end{array}$ & 3 & $6 \%$ \\
\hline $\begin{array}{l}\text { JOURNAL OF PRODUCTIVITY } \\
\text { ANALYSIS }\end{array}$ & 2 & $4 \%$ \\
\hline
\end{tabular}

Fuente: Elaboración propia.

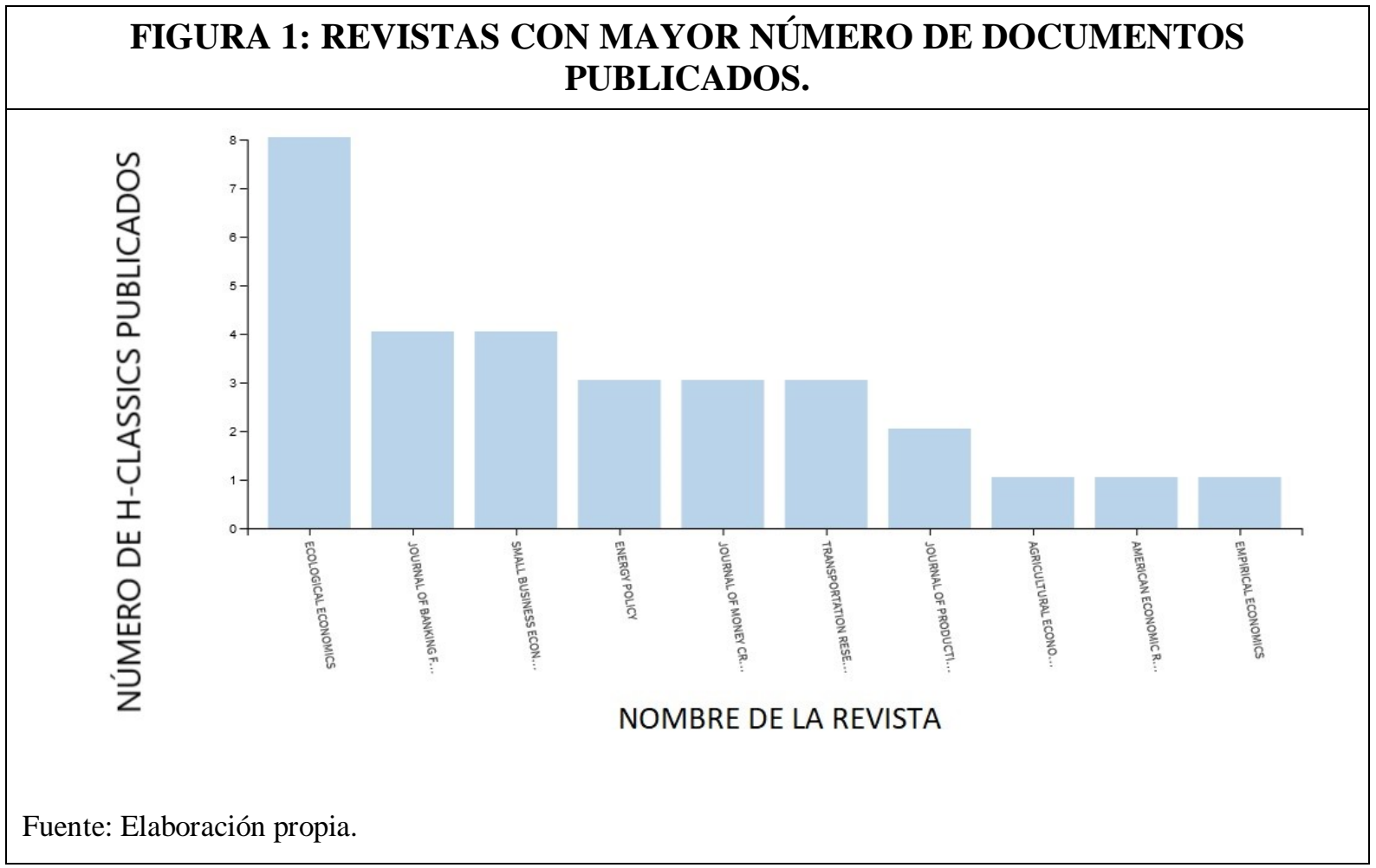




\section{3 Áreas de conocimiento relacionadas.}

Antes de comentar la tabla 4, es preciso realizar una explicación de la misma. Como se expone en la metodología, la búsqueda se realiza sobre la categoría o área temática de la Web of Science "Economics” y se filtran los resultados según lo ya explicado. Una vez seleccionados los 50 más citados, WoS permite a través de la herramienta “Analizar resultados” visualizar el ranking de áreas con más artículos H-Classics. Como cada uno de estos artículos puede estar publicado en una revista que pertenezca a varias áreas a la vez, se puede obtener la tabla 4. Obviamente todos ellos tienen que pertenecer al área Economics, por eso la fila correspondiente a este área se ha eliminado de la tabla.

Destacar en primer lugar Environmental Sciences Ecology, con casi tres veces más registros que la segunda y tercera áreas relacionadas, y con un destacable 26 por cien de cuota sobre todos los H-Classics. Destacar de nuevo su relación con la revista que más artículos HClassics posee publicados. Operations Research Management Science y Transportation que obtienen cinco registros cada una se colocan en segundo lugar. A raiz de esta tabla y de la anterior, un investigador del área de económicas se puede hacer una idea de cuáles son las áreas relacionadas más provechosas y dinámicas y qué revistas son punteras en estos ámbitos y decidir, a raíz de estos datos el futuro de sus investigaciones.

\begin{tabular}{l} 
TABLA 4: ÁREAS DE INVESTIGACIÓN RELACIONADAS. \\
\begin{tabular}{|l|l|l|}
\hline Área de investigación relacionada & Número de artículos & $\mathbf{\%}$ de $\mathbf{5 0}$ \\
\hline ENVIRONMENTAL SCIENCES ECOLOGY & 13 & $26 \%$ \\
\hline $\begin{array}{l}\text { OPERATIONS RESEARCH MANAGEMENT } \\
\text { SCIENCE }\end{array}$ & 5 & $10 \%$ \\
\hline TRANSPORTATION & 5 & $10 \%$ \\
\hline ENGINEERING & 4 & $8 \%$ \\
\hline $\begin{array}{l}\text { MATHEMATICAL METHODS IN SOCIAL } \\
\text { SCIENCES }\end{array}$ & 4 & $8 \%$ \\
\hline ENERGY FUELS & 3 & $6 \%$ \\
\hline AGRICULTURE & 2 & $4 \%$ \\
\hline
\end{tabular} \\
\hline
\end{tabular}

\subsection{Número de H-Classics publicados por año.}

Una vez obtenidos los 50 artículos más citados, mostramos en la figura 2 y en la tabla 5, la distribución de su publicación anual. Queremos destacar que ningún artículo considerado HClassics se ha publicado con anterioridad a 1995. De hecho, el 90 por cien de los H-Classics se publicaron a partir del año 2002. Igualmente, ningún artículo publicado a partir del año 2017 ha obtenido esa distinción, además de por otros posibles motivos, porque su ventana de citación ha sido más pequeña y quizás no ha tenido el tiempo suficiente para ser altamente citado. Los años con más H-Classics son el 2008 y el 2010 con 7 documentos cada uno. El periodo más fructífero de publicaciones H-Classics es el comprendido entre el año 2004 y 2010, con un 60 por cien de los H-Classics publicados. Los primeros $\mathrm{H}$-Classics publicados son los de Delcastillo y Benitez (1995a, 1995b).

Además, es reseñable que, como demuestra el gráfico, este es un campo que crece muy rápidamente, ya que, aunque normalmente los artículos más citados suelen haberse publicado en el siglo pasado, no ocurre lo mismo en este estudio. Incluso se observan cuatro artículos muy 
citados (De Ona et al., 2013; Faria et al., 2014; Iniesta-Arandia et al., 2014; Cansino et al., 2016), dentro del 1 por cien de artículos más citados para el campo, según la definición de la WoS (Clarivate Analytics, 2019), desde septiembre de 2013 a febrero de 2016.

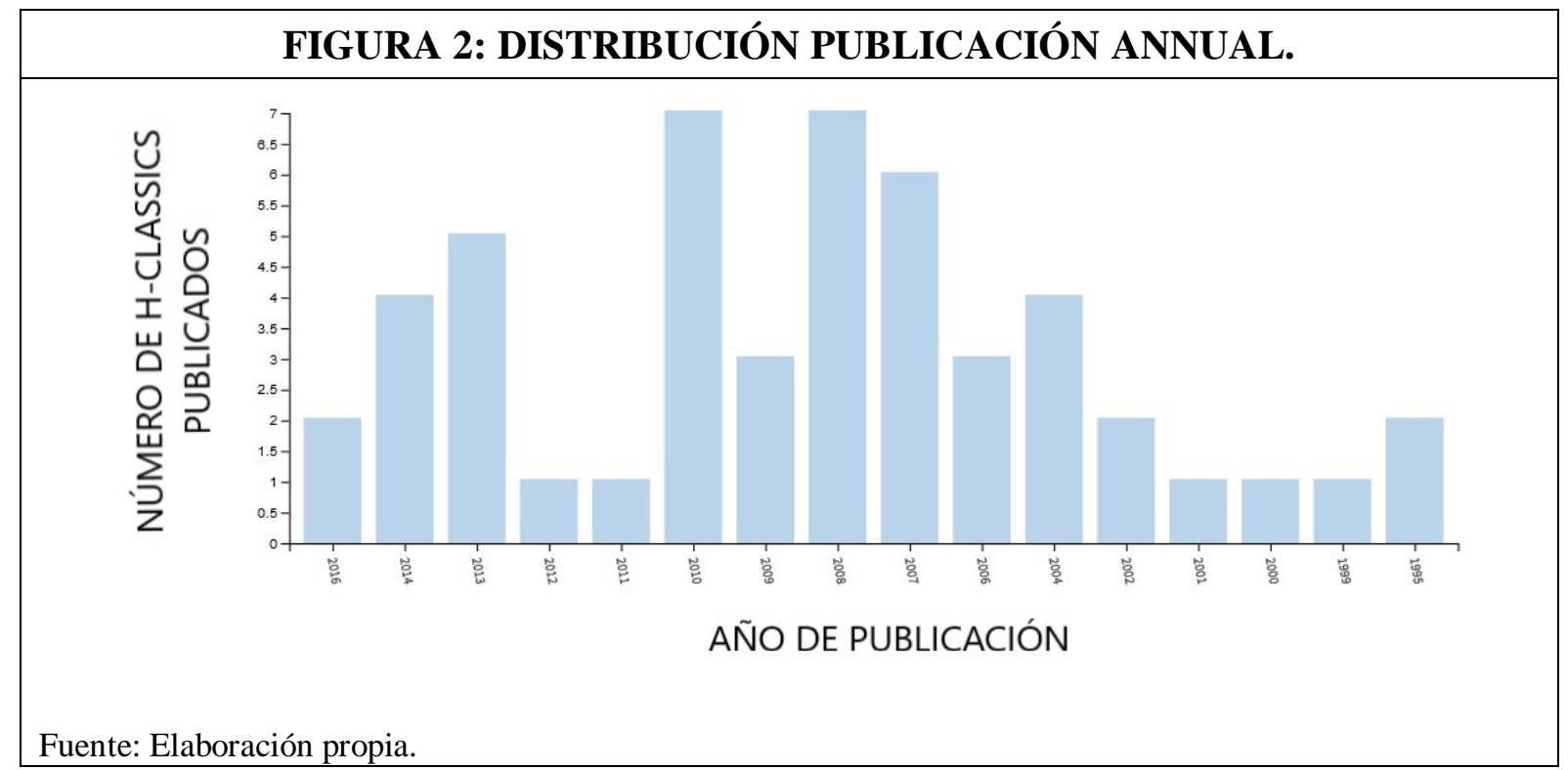

TABLA 5: DISTRIBUCIÓN PUBLICACIÓN ANUAL

\begin{tabular}{|l|l|l|}
\hline Años de publicación & Número de artículos & \% de 50 \\
\hline 2016 & 2 & $4 \%$ \\
\hline 2014 & 4 & $8 \%$ \\
\hline 2013 & 5 & $10 \%$ \\
\hline 2012 & 1 & $2 \%$ \\
\hline 2011 & 1 & $2 \%$ \\
\hline 2010 & 7 & $14 \%$ \\
\hline 2009 & 3 & $6 \%$ \\
\hline 2008 & 7 & $14 \%$ \\
\hline 2007 & 6 & $12 \%$ \\
\hline 2006 & 3 & $6 \%$ \\
\hline 2004 & 4 & $8 \%$ \\
\hline 2002 & 2 & $4 \%$ \\
\hline 2001 & 1 & $2 \%$ \\
\hline 2000 & 1 & $2 \%$ \\
\hline 1999 & 1 & $2 \%$ \\
\hline 1995 & 2 & $4 \%$ \\
\hline
\end{tabular}




\section{andaluzas.}

3.5 Países más productivos según su publicación de H-Classics con las universidades

La tabla 6 muestra el ranking de países con más artículos publicados H-Classics. Como todos los artículos de este estudio provienen de una institución española por la búsqueda y filtrado realizado, se ha eliminado de la tabla la fila de España por carecer de interés. Esta información se saca del campo Address, dependiente de cada autor. En primer lugar, y con más del triple de documentos que los segundos países en la lista, aparecen los Estados Unidos con diez documentos y, por tanto, un 20 por cien de los H-Classics. En segundo lugar, aparecen Inglaterra y Holanda con tres documentos. En esta tabla se observa que no hay ningún país hispanohablante y, por el contrario, copan los dos primeros puestos los países angloparlantes; por ello, parece recomendable publicar en estos últimos para tener más posibilidades de éxito.

\begin{tabular}{|l|l|l|}
\hline \multicolumn{3}{|c|}{ TABLA 6: PAÍSES CON MÁS ARTÍCULOS PUBLICADOS. } \\
\begin{tabular}{|l|l|l|}
\hline Países/Regiones & Número de artículos & $\%$ de 50 \\
\hline USA & 10 & $20 \%$ \\
\hline ENGLAND & 3 & $6 \%$ \\
\hline NETHERLANDS & 3 & $6 \%$ \\
\hline FRANCE & 2 & $4 \%$ \\
\hline GERMANY & 2 & $4 \%$ \\
\hline ITALY & 2 & $4 \%$ \\
Fuente: Elaboración propia. & \multicolumn{2}{|l}{} \\
\hline
\end{tabular}
\end{tabular}

\subsection{Universidades andaluzas que más H-Classics han publicado.}

Gracias a que en la WoS se incluye para cada artículo el campo address donde aparece la institución a la que pertenece el autor; se pueden identificar las instituciones que más H-Classics han publicado, lo que se muestra en la tabla 7. En esta tabla mostramos solamente las universidades andaluzas; posteriormente, enseñaremos las universidades españolas y extranjeras en las que más se colabora en este ámbito.

TABLA 7: UNIVERSIDADES ANDALUZAS CON MÁS ARTÍCULOS
PUBLICADOS.
\begin{tabular}{|l|l|l|}
\hline Nombre institución & Número de artículos & $\%$ de 50 \\
\hline UNIVERSIDAD DE GRANADA & 12 & $24 \%$ \\
\hline UNIVERSIDAD DE SEVILLA & 12 & $24 \%$ \\
\hline UNIVERSIDAD PABLO DE OLAVIDE & 11 & $22 \%$ \\
\hline UNIVERSIDAD DE MALAGA & 10 & $20 \%$ \\
\hline UNIVERSIDAD DE CORDOBA & 3 & $6 \%$ \\
\hline UNIVERSIDAD DE ALMERIA & 2 & $4 \%$ \\
\hline UNIVERSIDAD DE HUELVA & 2 & $4 \%$ \\
\hline UNIVERSIDAD DE JAEN & 1 & $2 \%$ \\
\hline Fuente: Elaboración propia. & \multicolumn{2}{c|}{} \\
\hline
\end{tabular}


En esta tabla se pueden observar tres grupos de universidades andaluzas claros. Por un lado, las Universidades de Granada, Sevilla, Pablo de Olavide y Málaga que suman 45 documentos; por tanto, el 90 por cien de los H-Classics se sitúan a la cabeza en este ranking. Un segundo grupo de universidades con 7 documentos y un 16 por ciento del total, como son Córdoba, Almería, Huelva y Jaén, que necesitarían mejorar en este ámbito para acercarse a las 4 más potentes. Por último, tendríamos a la Universidad de Cádiz donde no se ha publicado ningún H-Classics hasta el momento. Hay que tener en cuenta que, si sumamos todos los porcentajes, saldría más que el 100 por cien, en concreto un 106 por cien, puesto que hay artículos que están firmados por investigadores de varias universidades andaluzas. Que el porcentaje sumado un tan escasamente superior al 100 por cien sugiere una escasa colaboración entre investigadores de las universidades andaluzas en este campo.

\subsection{Universidades españolas y extranjeras con las que más H-Classics se publican.}

En la tabla 8 se muestran las instituciones españolas y extranjeras en las que se han publicado 2 o más H-Classics; como se puede observar, hay tan sólo 3 instituciones con las que se han publicado 2 documentos, un número ciertamente escaso. Señalar que las dos instituciones extranjeras, Indiana University Bloomington y University of Pennsylvania, son de EEUU, mientras que la nacional es la Universidad de Valladolid.

Por razones de espacio no se añaden a este listado las instituciones españolas o extranjeras con las que se ha publicado un solo documento; son muy numerosas, lo que viene a indicar cierta colaboración, pero no suficientemente arraigada.

\section{TABLA 8: UNIVERSIDADES ESPAÑOLAS Y EXTRANJERAS CON MÁS ARTÍCULOS PUBLICADOS.}

\begin{tabular}{|l|l|l|}
\hline Nombre de la institución & Número de artículos & \% de 50 \\
\hline INDIANA UNIVERSITY BLOOMINGTON & 2 & $4 \%$ \\
\hline UNIVERSIDAD DE VALLADOLID & 2 & $4 \%$ \\
\hline UNIVERSITY OF PENNSYLVANIA & 2 & $4 \%$ \\
\hline
\end{tabular}

Fuente: Elaboración propia.

\section{CONCLUSIONES.}

Antes de pasar a las conclusiones hay que precisar que, aunque esta investigación muestra una visión general interesante sobre los $\mathrm{H}$-Classics de las universidades públicas andaluzas en la categoría Economics del JCR tiene algunas limitaciones. Al utilizar la base de datos de WoS solo se han analizado exclusivamente los documentos que están indexados en esta base de datos. Además, al escoger la categoría Economics del JCR sólo se están analizando los documentos de las revistas indexadas dentro de esa categoría, pudiendo quedar fuera documentos sobre la temática económica que habría sido de interés para este estudio.

En este estudio se ha utilizado como base los artículos pertenecientes a la categoría economics de la WoS; de ellos, se han tenido en cuenta solo los que tienen algún investigador firmante de una universidad andaluza. Sobre estos documentos se han analizado solamente los altamente citados, el H-Core de los mismos, utilizando el concepto de H-Classics, que está basado en el H-index. Se han encontrado los siguientes hallazgos:

-Se han identificado 50 artículos H-Classics en todo el periodo de tiempo que analiza la WoS, con citaciones que van entre las 50 y las 195 citas. El artículo más citado con cierta diferencia es el de Dietsch y Lozano-Vivas (2000). 
-Hay que destacar también el artículo De Ona et al. (2013) que ocupa el puesto 5 en el ranking, consiguiendo un número elevado de citas (128) habiéndose publicado en una fecha relativamente reciente, septiembre del año 2013 y por tanto con una ventana de citas corta.

-La catedrática Lozano-Vivas de la Universidad de Málaga, directora del grupo de investigación Fundamentos Microeconómicos, con 4 documentos, es la investigadora con más H-Classics y, por tanto, referencia en esta temática.

-La revista Ecological Economics, con el doble de artículos publicados que la segunda revista, y el área de conocimiento relacionada ENVIRONMENTAL SCIENCES ECOLOGY como área donde más H-Classics se publican, son los ámbitos donde más dinamización se observa dentro de la categoría Economics y un buen nicho de trabajo al que dedicarse en el futuro.

-En cuanto al patrón temporal de publicación de los H-classics, este es un campo que está creciendo muy rapidamente, donde los artículos mas citados son casi todos del siglo XXI. Incluso hay 4 artículos altamente citados dentro de la categoría, donde se pone en relación el número de citas con un tiempo corto en conseguirlas, lo cual habla de la buena salud de la investigación andaluza en este ámbito.

-Destacar la predominancia de EEUU en cuanto a los paises con los que más se colabora para la publicación de artículos altamente citados, así como con las universidades estadounidenses de Indiana y Pennsylvania. Hay que señalar que hay colaboraciones con el extranjero pero, en lo que a este estudio se refiere, todavía débiles.

-En el caso de las Universidades de Andalucía se puede observar la existencia de una Andalucía de "dos velocidades" en este ámbito concreto de investigación. Las instituciones de mayor envergadura, como son las universidades de Granada, Málaga, Sevilla y Pablo Olavide, sacan mucha ventaja a las universidades más pequeñas de ciudades como Córdoba, Huelva, Almería, Jaen y Cádiz.

Por último, hay que mencionar que la información proporcionada en este estudio puede ser de gran utilidad para comprender la estructura científica pasada, presente y futura dentro del ámbito de la economía en las universidades y, por tanto, podría ayudar al futuro desarrollo de la investigación.

\section{BIBLIOGRAFÍA.}

Alonso, S., Cabrerizo, F. J., Herrera-Viedma, E., Herrera, F. (2009): “H-Index: A review focused in its variants, computation and standardization for different scientific fields". Journal of Informetrics, vol. 3, nº 4, 273-289. https://doi.org/10.1016/j.joi.2009.04.001

Baltussen, A., Kindler, C. (2004): “Citation classics in critical care medicine”. Intensive Care Med, no 30, pp. 902-910.

Cabrerizo, F. J., Martínez, M. A., Cobo, M. J., Lázaro-Rodríguez, P., López-Gijón, J. y HerreraViedma, E. (2017): “Aggregation operators in group decision making: Identifying citation classics via H-classics”. Procedia computer science, $\mathrm{n}^{0}$ 122, pp. 902-909.

Cansino, J. M., Roman, R. y Ordonez, M. (2016): "Main drivers of changes in CO2 emissions in the Spanish economy: A structural decomposition analysis”. Energy Policy, ${ }^{\circ}{ }^{\circ}$ 89, pp. 150-159. https://doi.org/10.1016/j.enpol.2015.11.020

Cassar, A., Downey, R., Byrne, D., Molony, D. y Mulhall, K. (2012): "The 25 most cited articles in arthroscopic orthopaedic surgery”. Arthroscopy, vol. 28, n ${ }^{\circ} 2$, pp. 548-564.

Clarivate Analytics. (2019): “Colección principal de Web of Science Ayuda”. http://images.webofknowledge.com/WOKRS522_2R1/help/es_LA/WOS/hs_citation_ applications.html Consultado el 30 de Marzo de 2020. 
Chiang, H. S., Huang, R. Y., Weng, P. W., Mau, L. P., Tsai, Y. W., Chung, M. P., ... y Cheng, W. C. (2018): "Prominence of scientific publications towards peri-implant complications in implantology: A bibliometric analysis using the H-classics method". Journal of oral rehabilitation, vol. 45, $\mathrm{n}^{\circ}$ 3, pp. 240-249.

Delcastillo, J. M. y Benitez, F. G. (1995a): "On the functional form of the speed-density relationship .1. General-theory”. Transportation Research Part B-Methodological, vol. 29, no 5, pp. 373-389.

Delcastillo, J. M., y Benitez, F. G. (1995b): "On the functional form of the speed-density relationship .2. Empirical-investigation”. Transportation Research Part BMethodological, vol. 29, n ${ }^{\circ}$ 5, pp. 391-406.

De Ona, J., de Ona, R., Eboli, L. y Mazzulla, G. (2013): "Perceived service quality in bus transit service: A structural equation approach”. Transport Policy, $\mathrm{n}^{\mathrm{0}}$ 29, pp. 219-226. https://doi.org/10.1016/j.tranpol.2013.07.001

Dietsch, M., y Lozano-Vivas, A. (2000): "How the environment determines banking efficiency: A comparison between French and Spanish industries”. Journal of Banking \& Finance, vol. 24, no 6, pp. 985-1004. https://doi.org/10.1016/s0378-4266(99)00115-6.

Faria, R., Gomes, M., Epstein, D., y White, I. R. (2014): “A Guide to Handling Missing Data in Cost-Effectiveness Analysis Conducted Within Randomised Controlled Trials". Pharmacoeconomics, vol. 32, no 12, pp. 1157-1170. https://doi.org/10.1007/s40273014-0193-3.

Feijoo, J., Limeres, J., Fernández-Varela, M., Ramos, I. y Diz, P. (2013): “The 100 most cited articles in dentistry”. Clinical Oral Investigations, $\mathrm{n}^{\circ}$ 18, pp. 699-706.

Garfield, E. (1977): "Introducing citation classics. The human side of scientific reports," Current Comments, vol. 1, pp. 5-7.

Garfield, E. (1987): "100 citation classics from the Journal of the American Medical Association,” Journal of the American Medical Association, vol. 257, nº 1, pp. 52-59.

Gehanno, J., Takahashi, K., Darmoni, S. y Weber, J. (2007): "Citation classics inoccupational medicine journals”. Scandinavian Journal of Work, Environ \& Health, vol. 33, n 4, pp. $245-251$.

Harzing, A., van der Wal, R. (2008): “Google scholar as a new source for citation analysis”. Ethics in Science and Environmental Politics, $\mathrm{n}^{\circ}$ 8, pp. 61-73. doi:10.3354/esep0007

Hirsch, J. (2005): “An index to quantify an individual's scientific research output”, Proc. Nat. Academy Sci., vol. 102, no 46 pp. 16569-16572.

Hui, J., Han, Z., Geng, G., Yan, W. y Shao, P. (2013): "The 100 top-cited articles in orthodontics from 1975 to 2011”. Angle Orthodontist, vol. 83, n³, pp. 491-499.

Ibrahim, G., Snead, O., Rutka, J. y Lozano, A. (2012): "The most cited works inepilepsy: Trends in the "citation classics"”. Epilepsia, vol. 53, n 5, pp. 765-770.

Iniesta-Arandia, I., Garcia-Llorente, M., Aguilera, P. A., Montes, C., y Martin-Lopez, B. (2014): "Socio-cultural valuation of ecosystem services: uncovering the links between values, drivers of change, and human well-being”. Ecological Economics, vol. 108, pp. 36-48. https://doi.org/10.1016/j.ecolecon.2014.09.028

Levitt, J. y Thelwall, M. (2009): “The most highly cited library and information science articles: Interdisciplinarity, first authors and citation patterns”. Scientometrics, vol. 78, $\mathrm{n}^{\circ} 1$, pp. 45-67.

Martínez, M. A., Cobo, M. J., Herrera, M., Herrera-Viedma, E. (2015): “Analyzing the scientific evolution of social work using science mapping”. Research on Social Work Practice, vol. 25, $\mathrm{n}^{\circ}$ 2, pp. 257-277.

Martínez, M. A., Herrera, M., Contreras, E., Ruíz, A. y Herrera-Viedma, E. (2014a): "Characterizing Highly Cited Papers in Social Work Through H-Classics". Scientometrics, $\mathrm{n}^{\mathrm{o}}$ 102, pp. 1713-1729. 
Martínez, M. A., Herrera, M., López-Gijón, J., Herrera-Viedma, E., (2014b): "H-classics: Characterizing the concept of citation classics through h-index”. Scientometrics, $\mathrm{n}^{\circ}$ 98, pp. 1971-1983.

Perez-Cabezas, V., Ruiz-Molinero, C., Carmona-Barrientos, I., Herrera-Viedma, E., Cobo, M. J., y Moral-Munoz, J. A. (2018): "Highly cited papers in Rheumatology: Identification and conceptual analysis”. Scientometrics, vol. 116, n ${ }^{\circ}$ 1, pp. 555-568.

Ponce, F., Lozano, A. (2010): "Highly cited works in neurosurgery. part ii: the citation classics”. Journal of Neurosurgery, vol. 112, n 2, pp. 233-246.

Ponce, F., Lozano, A., (2011): “The most cited works in parkinson's disease”. Movement Disorders, vol. 26, n 3, pp. 380-390.

Rosenberg, A., Tripathi, R. y Blum, J. (2010): “The most influential articles in critical care medicine”. Journal of Critical Care, $\mathrm{n}^{\circ}$ 25, pp. 157-170.

Seglen, Per O. (1997) "Why the impact factor of journals should not be used for evaluating research”. British medical journal, vol. 314, pp. 498-502. https://doi.org/10.1136/bmj.314.7079.497

Smith, D. (2007): “Ten citation classics from the New Zealand medical journal.” New Zealand Medical Journal, vol. 120, nº. 1267, pp. 2871-2875.

Stack, S. (2012): “Citation classics in suicide and life threatening behavior: A research note,”. Suicide Life-Threatening Behav, vol. 42, nº. 6, pp. 628-639.

Stack, S., (2013): “Citation classics in deviant behavior: A research note”. Deviant Behavior, vol. 34, no 2, pp. 85-96.

Tam, W., Wong, E., Wong, F. y Cheung, A. (2012): "Citation classics in the integrative and complementary medicine literature: 50 frequently cited articles”. European Journal of Integrative Medicine, $\mathrm{n}^{\circ}$ 4, pp. e77-e83.

Tam, W., Wong, E., Wong, F. y Hui, D. (2013): "Citation classics: Top 50 cited articles in 'respiratory system””. Respirology, vol. 18, nº. 1, pp. 71-81. 